\title{
Inside information in Ponzi schemes
}

\author{
Klarita Sadiraj $^{1} \cdot$ Arthur Schram $^{2,3}$
}

Received: 20 December 2016 / Revised: 3 May 2018 / Accepted: 7 May 2018 /

Published online: 18 May 2018

(C) The Author(s) 2018

\begin{abstract}
Ponzi-like investment schemes were popular in many transition economies. Often, some government officials had inside information about the viability of such schemes and used this information to their own advantage. We introduce a novel experimental design that allows us to study the extent to which this kind of abuse of information is possible and what consequences it has for those without such information. In particular, we investigate how the proportion of informed versus uninformed investors and the promised dividends affect the way in which informed investors can exploit the investments of uninformed investors. Our results show that uninformed investors follow the observed choices of the informed even more than predicted by theory. This adds to the devastating effects that this kind of underground activity can have on the uninformed.
\end{abstract}

Keywords Ponzi scheme $\cdot$ Informed investors $\cdot$ Laboratory experiment

JEL classification C $92 \cdot$ P 39

We are grateful to Utpal Bhattacharya, Govert Bijwaard, Jim Cox, Vjollca Sadiraj, Sweder van Wijnbergen for useful comments.

Electronic supplementary material The online version of this article (https://doi.org/10.1007/ s40881-018-0047-4) contains supplementary material, which is available to authorized users.

Arthur Schram

schram@uva.nl

1 The Netherlands Institute for Social Research (SCP), Den Haag, The Netherlands

2 Department of Economics and Econometrics, CREED, Amsterdam School of Economics, Roetersstraat 11, 1018 WB Amsterdam, The Netherlands

3 Robert Schumann Center, European University Institute, Florence, Italy 


\section{Introduction}

Many transition economies witnessed the rise and fall of lucrative investment opportunities offering spectacular dividends (van Brabant 1998). Though often based on valuable resources, the returns to investment were vastly overrated and dividends could only be covered by new investments. This characterizes such investment funds as Ponzi schemes. ${ }^{1}$ Such schemes are illegal and these investment opportunities were mostly observed in underground economies. When a scheme collapsed (as they all inevitably do), there was then no legal framework to support those who had lost their investments.

The important thing that all Ponzi schemes have in common is that to survive, they need to use invested funds to pay other investors. When there is insufficient money left (e.g., because investors start to withdraw), they collapse. The Ponzi schemes we are interested in typically involve the development of an overvalued resource such as oil, gas, minerals or real estate. The promoter convinces investors that the asset can be further developed with more capital, and the promoter will share the profits with the investors. Typically, substantial dividends are paid early on. The representation is that these dividends are 'profits' coming from the successful development. What is actually happening is that the promoter is merely returning a portion of the investors' money to them. These early and substantial dividends attract additional investors and induce early investors to increase their share. For the scheme to survive, the amount invested must exceed the amount needed for dividends.

A particular characteristic in some transition countries (most notably Albania in 1997) aggravated the negative impact of these schemes (Sadiraj 1999; Shala 1997; Gërxhani and Schram 2009). This is that certain members of the government had inside information about the scheme's viability. Instead of stopping the process early on, the common view is that they used this information to their own advantage by investing in the fund and getting out 'on time'. The extent to which this type of inside information can be used to one's own benefit and the consequences for the large group of uninformed investors are unknown, however. This paper addresses this issue.

For this purpose, we consider a Ponzi-scheme environment with two types of investors: informed and uninformed (Sadiraj et al. 1997). In the 1997 Ponzi schemes

\footnotetext{
1 In 1920, the Italian-born immigrant Charles Ponzi offered investors in Massachusetts a 50\% return on their investment in 90 days. He was using the money invested by others to pay out interest. The system collapsed after 10,000 people had invested almost ten million dollars. The term 'Ponzi-schemes' is now used to describe games where individuals or companies pay out funds to some parties by borrowing funds from others. Though the term 'pyramid scheme' is often used for this type of game, it should be noted that they are a specific kind of Ponzi scheme. First, a pyramid scheme involves investing for the right to receive compensation for introducing new participants. There is a clear understanding that success depends on attracting additional participants. In a non-pyramid Ponzi scheme (like the one by Ponzi himself), participants believe that success depends on the development of some productive asset. Second, pyramids must fail because their success depends on endless exponential participation growth. Other Ponzi schemes eventually fail because the underlying asset either never existed, or was grossly overvalued. Contrary to pyramid schemes, other Ponzi schemes can flourish even with passive investors.
} 
in Albania, for example, informed investors were governmental employees trying to exploit the influence they had. Informed investors control the main sources of information dissemination, the media. They give positive information about the schemes suggesting, for example, government support. Their informational advantage allows informed investors to withdraw from the scheme on time.

It is very difficult to obtain observational field data on Ponzi schemes. Aside from the fact that many are illegal and, therefore, do not have public records, the records that are available generally have very noisy data. They have typically flourished in countries, where reliable data on variables like inflation or interest rates are difficult to find. Laboratory control allows one to circumvent these problems. Therefore, to better understand individual behavior in such schemes, we develop a novel laboratory experiment. We observe the behavior and decisions of individuals in an experimental investment project with the main characteristics of Ponzi schemes. These include an unrealistically high-interest rate, the possibility of keeping the scheme alive using invested funds to pay interest and an increasing probability of bankruptcy as time passes. In addition, as in Sadiraj et al. (1997), we distinguish between informed from uninformed investors. Our controlled Ponzi scheme allows for a real return to investments. This return may be insufficient to cover the interest paid, however. Only the informed investors know the real return. Our results show that Ponzi schemes can easily be generated in the laboratory. The duration of participation is unaffected by the aggregate number of investors, decreases with the relative number of informed investors, and decreases with the interest rate.

As far as we know, Ponzi schemes have not been studied in a laboratory setting before. There are two closely related phenomena that have been studied experimentally, however. The first are bubbles in financial markets. These have many characteristics similar to Ponzi schemes. Rationally, they should not occur. Nevertheless, it is generally believed that they do, and there is a vast theoretical literature trying to explain why (e.g., Brock 1982; Tirole 1985; O'Connell and Zeldes 1988; Gilles and LeRoy 1992). In addition, bubbles have been observed in laboratory markets (e.g., Smith et al. 1988; King 1993; Sunder 1995). Our paper adds to this literature by showing that Ponzi schemes are observed in the laboratory as well. The secondrelated phenomenon are so-called bank runs (Shotter and Yorulmazer 2009; Brown et al. 2012). Like Ponzi schemes, large financial institutions are in danger of collapsing if too many investors simultaneously want to withdraw their investments. An important difference is that Ponzi schemes need invested money to pay interest and will eventually collapse even if no investor withdraws.

\section{The Ponzi game}

In the game investors decide whether or not to invest a fixed sum $Y$ (equal across investors) in an investment fund (IF). Investors $j \in I \cup U$ are either informed $(j \in I)$ or uninformed $(j \in U)$. We use the notation ' $I$ ' (' $U$ ') both to denote the set of informed (uninformed) investors and the cardinality of these sets. The total number of (potential) investors is $N=I+U$. 
Before individual investment decisions are made $(t=0)$, there is an initial investment $\chi$ in IF, reflecting real returns from a valuable underlying resource. We assume that nature draws $\chi$ from a known set of integers between $p$ and $q$, with equal probabilities, i.e., $\chi \in\{p, \ldots, q\}$, with $q>p>0$. In period 1 , informed investors are told the realization of $\chi$. They either invest $Y$ in IF or invest nothing, giving an action space $\{0, Y\}$. As long as bankruptcy does not occur (see below), the game moves on to the uninformed.

There is a restriction in the actions allowed. If an investor has previously withdrawn (i.e., she has invested zero after previously investing $Y$ ), she is not allowed to reinvest. This means that withdrawal is final. As will become clear, this restriction simplifies the analysis considerably. As a consequence, the action space in later periods depends on previous choices. Having invested in period $t$, the investor must choose again in $t+1$ whether to invest (choose " $Y$ ") or withdraw (choose " 0 "). Those who have not previously invested or have invested in $t-1$ thus face the same action space $\{0, Y\}$. Those who have invested 0 in period $t_{2}$ after investing $Y$ in $t_{1}<t_{2}$ (that is, they have 'withdrawn' a previous investment) can no longer invest and face action space $\{0\}$. In short, players can invest in period 1, or enter in a later period conditional on not having withdrawn an earlier investment. An investment can be withdrawn in any period as long as no bankruptcy has occurred. Finally, note that the fact that the players can only invest a fixed amount $(Y)$ and cannot add to the investment in later periods is aimed at simplifying the environment for the theoretical analysis and experimental implementation. We leave such further options for future research.

The uninformed players do not know the realization of $\chi$ but know that $\chi$ is a stochastic variable with known distribution. After the informed have made their decision in a period, and if bankruptcy does not occur, the uninformed choose a strategy from $\{0, Y\}$. If bankruptcy does not occur (see below), an interest payment $\pi_{j}=d_{j} r Y$ is subsequently paid to every $j \in I, U$, where $d$ is a dummy denoting whether or not $j$ is invested in IF and $r$ is the interest rate. Interest payments are paid out of IF and diminish the amount available for the future. If the amount of interest previously paid is larger than $\chi$, then the money in IF is insufficient to pay back the investments to all investors, should they simultaneously withdraw. This makes the game a Ponzi scheme.

In any period, the informed have the option of withdrawing their funds before the uninformed make their decisions. The decisions by the informed are not made public until the end of the period. At that point, the aggregate investment decisions per type (informed and uninformed) are announced and a new period starts. Thus, when the informed make their decision, they know what the (aggregate) most recent decision of the uninformed is (and, knowing the realization of $\chi$, they know the exact amount of funds in IF). On the other hand, the uninformed know neither the realization of $\chi$, nor the most recent investment decisions of the informed when they decide what 
to do. ${ }^{2}$ This is because the uninformed make a decision in $t$, after the informed have already done so. At that point, they know only all events up to the end of $t-1$.

In every period, there are two points in time when bankruptcy may occur. First, when the informed make their investment decision, some might want to withdraw. If the amount they wish to withdraw is less than the funds available in IF, the withdrawals are realized. If not, bankruptcy occurs. Second, at the end of the period, money is needed to pay for the withdrawals of the uninformed participants plus all interest payments. If there is enough money available in IF, these are realized. If not, bankruptcy occurs. In case of bankruptcy, the funds remaining in IF are equally split across all remaining investors. ${ }^{3}$ ESM Appendix A provides an overview of the time flow of the game.

Note that the uninformed in our game know that they are involved in a Ponzi scheme; they do not know, however, when it will collapse. For the experimental design, this is a choice made to avoid deceiving participants. The extent to which this harms the external validity of our results depends on whether or not investors outside the laboratory suspect that they are in a Ponzi scheme (but hope to benefit and withdraw on time). Given the nature of these schemes, it is unlikely that one would be able to elicit such suspicions in the field. ${ }^{4}$

\section{Equilibrium investment}

First, consider efficiency. Note that the initial investment $\chi$ (the 'real return') can only be realized (earned by the subjects) if sufficient investments are made. Any (interest) earnings exceeding $\chi$ are effectively a redistribution of income. Therefore, any outcome where there are sufficient investments to have $\chi$ paid out as interest is efficient.

In this multi-stage game, a strategy is a complete plan of action. To start, consider the case, where every player keeps $Y$ invested in every period. Then, bankruptcy occurs and the remaining funds are distributed evenly. Each investor earns $Y+\chi / N$. ESM Appendix A shows that this is generally not an equilibrium (it is not an equilibrium for the parameters used in our experiments). Similarly, no outcome can be an equilibrium if all investments are withdrawn and more than $r Y$ is left in IF (because a single investor can increase her earnings by investing one more period).

In ESM Appendix A, we derive a Bayesian-Nash equilibrium for the game in quasi-symmetric strategies, i.e., symmetric strategies within the group of informed traders and symmetric strategies within the group of uninformed traders. This equilibrium is characterized by three parameters $t_{p} \leq t_{\chi} \leq t_{q}$, defined as the maximum

\footnotetext{
${ }^{2}$ In Ponzi schemes outside the laboratory, the uninformed may or may not know the number of investors (informed and uninformed) in periods without bankruptcy. The key characteristic is that the uninformed can only find out too late that the informed withdrew. Our two-stage framework within each period allows us to capture this characteristic in a simple manner.

${ }^{3}$ Note that the interest rate, $r$, may affect the timing of bankruptcy (the lower is $r$, the longer IF can sustain interest payments), but not its consequences.

${ }^{4}$ We thank an anonymous reviewer for pointing this out.
} 
integer number of periods in which interest can be paid to all investors, using only the amount $p, \chi, q$, respectively.

In this equilibrium, the following strategies are used. The uninformed start investing in period 1 . The number of investment periods that maximizes their expected earnings is denoted by $t^{U}$ and is the integer in $\left[t_{p}, t_{q}\right]$ closest to $\frac{I}{N} t_{p}+\frac{U}{N} t_{q}+\frac{(U-I)}{2 N}>t_{p}$. The uninformed withdraw in period $t^{U}+1$, unless they observe at least one informed investor withdrawing in $t^{*}<t^{U}$, in which case they withdraw in $t^{*}+1$ (recall that $j \in U$ does not know that $j \in I$ has withdrawn until $t^{*}$ has been completed).

The informed start investing in period 1 . They withdraw in $t^{U}$ if the realized draw of $\chi$ is insufficient to pay interest to all investors for $t^{U}$ periods. Otherwise, they invest as long as $\chi$ is sufficient to pay interest to all investors until $t^{U}$ and only the informed in $t>t^{U}$. If some $j \in U$ withdraw before $t^{U}$, the informed stay in as long as $\chi$ is sufficient to pay interest to all remaining investors.

This equilibrium is quite intuitive. The uninformed determine their optimal duration of investment, taking into account that the informed may take advantage of them. Unless the informed do something out of equilibrium (like withdraw unexpectedly early), their choices are not affected by what the informed do. The intuition underlying the behavior of the informed is that they will withdraw at the last chance before the uninformed do if the latter are 'overestimating' the amount of money available. In this case, the informed will draw interest from the investments by the uninformed. If the uninformed are underestimating the amount available, the informed will stay in until $\chi$ is (almost) depleted.

Finally, we note that with risk-neutral players there can be no equilibrium without interest being paid out of others' investments for at least some draws of $\chi$. To see this, first note that all uninformed will stay in longer than $t_{p}$ periods. This is because doing so is dominated by staying in until $t^{U}>t_{p}$. Then, the informed withdrawing in period $t_{p}+1$ weakly dominates withdrawing in $t_{p}$ and they will stay in longer than $t_{p}$ periods. This means that for $\chi=p$ they will draw interest from the investments by the uninformed, which creates a Ponzi situation.

\section{Procedures and parameters}

The experiments were run at the CREED laboratory of the University of Amsterdam. Subjects were recruited from the undergraduate population. In total, 224 subjects participated. This experiment lasted about $1 \mathrm{~h}$. On average, participants earned the equivalent of $\$ 17$.

In all sessions, $Y=250$. We used a complete $2 \times 2 \times 2$ design, varying the number of participants $(N=16$ versus $N=12)$, the interest rate $r(r=0.1$ versus $r=0.2)$, and the relative number of informed, $(I=1, U=N-1$ versus $I=U=N / 2$ ). The boundary values $p$ and $q$ depend on the number of participants. For $N=12$, we chose $p=1200, q=3600$ and for $N=16$, the values are proportionally higher: $p=1600, q=4800$. We shall refer to the high (low) interest sessions as $\mathrm{Hi}$ (Lo) and to the sessions with 1 informed subject(s) as 1 . We will refer to sessions $(N=12$ or $N=16)$ with half of the subjects informed by " $8(6)$ ". Because we ran a full factorial between-subject design, we have eight treatments: 
Table 1 Realized random draws of $\chi$

\begin{tabular}{lrrrrrrrr}
\hline Round & \multicolumn{1}{c}{ 1 } & \multicolumn{1}{c}{3} & \multicolumn{1}{c}{3} & \multicolumn{1}{c}{4} & 5 & 6 & 7 & 8 \\
\hline$\chi$ & 2400 & 4800 & 1600 & 1600 & $3600^{\mathrm{a}}$ & 4000 & $2000^{\mathrm{b}}$ & $3600^{\mathrm{a}}$ \\
$t_{\chi}$ (low interest) & 6 & 12 & 4 & 4 & 9 & 10 & 5 & 9 \\
$t_{\chi}$ (high interest) & 3 & 6 & 2 & 2 & 4 & 5 & 3 & 4 \\
\hline
\end{tabular}

a3200 in the high-interest sessions (see fn. 5)

b2400 in the high-interest sessions (see fn. 5)

Hi 1 - 12; Hi 8 - 12; Lo 1 - 12; Lo 8 - 12; Hi 1 - 16;Hi 8 - 16; Lo 1 - 16; Lo 8 - 16. We ran each session twice, for a total of 16 sessions. In each session, 8 different investment funds were run in 8 consecutive rounds. Each round continued until either there were no more investments or the investment fund was bankrupt.

The order of events and information flows in the experiment follow closely the model presented above. A translation of the instructions is provided in ESM Appendix C. To minimize noise, the realized draws of $\chi$ were made once for all sessions. They were reported to (only) the informed at the start of a round. We adapted the numbers across sessions to account for the distinct parameter configurations. We adapted $\chi$ such, that $t_{\chi}$ (the number of periods with enough 'outside' money to pay interest to all participants) was comparable across sessions. For example, in round 1 of sessions with $N=16$ and $r=0.1, \chi=2400$ was used. Then, 400 is paid in interest in each period when all participants invest; ergo, $t_{\chi}=6$. To adapt for $N=12$, we used $\chi=0.75 \times 2400=1800$. Here, 300 per period is paid in interest and once again $t_{\chi}=6$. For $r=0.2,800(N=16)$ or $600(N=12)$ is paid per period when everyone invests, implying $t_{\chi}=3$. To avoid income effects, we did not adjust $\chi$ between the low- and high-interest sessions. We argue below that a simple rescaling of time makes the sessions with high and low interest rates comparable. ${ }^{5}$ Table 1 gives an overview of the realized random draws of $\chi$ in the 8 rounds and the corresponding $t_{\chi}$ values.

ESM Appendix B provides for each treatment an overview of the key variables that determine equilibrium behavior. These yield the following comparative static predictions:

1. The number of investors will not affect behavior.

2. An increase in the number of informed investors from 1 to 8(6) will decrease the duration of investment by the uninformed.

3. The duration of investment will be lower when $r=0.2$ than when $r=0.1$.

\footnotetext{
5 In some cases, the value drawn for $\chi$ was not dividable by 600 (800) and therefore not in the set used for the random draw in case of a high interest. In these cases we adjusted the value as described in the notes of Table 2 .
} 
Table 2 Median withdrawal period

\begin{tabular}{|c|c|c|c|c|c|c|c|c|}
\hline & \multicolumn{4}{|c|}{ Low interest, $r=0.1$} & \multicolumn{4}{|c|}{ High interest, $r=0.2$} \\
\hline & \multicolumn{2}{|l|}{$I=1$} & \multicolumn{2}{|l|}{$I=6 / 8$} & \multicolumn{2}{|l|}{$I=1$} & \multicolumn{2}{|l|}{$I=6 / 8$} \\
\hline & $N=12$ & $N=16$ & $N=12$ & $N=16$ & $N=12$ & $N=16$ & $N=12$ & $N=16$ \\
\hline Informed & 6 & 12 & 11 & 10 & 7 & 7 & 6 & 6 \\
\hline Uninformed & 12 & 12 & 10 & 10 & 7 & 7 & 6 & 6 \\
\hline
\end{tabular}

Cells give the median period of withdrawal across all participants and all rounds in the treatment combination concerned. Recall that the equilibrium withdrawal period with high interest is half the equilibrium period with low interest. The numbers for the informed in cases with only one informed are based on only two individuals each, each with eight decisions

\section{Experimental results}

\subsection{General results}

A first question is whether the Ponzi schemes in the laboratory collapse like they do in the outside world. The answer is a clear 'yes'. In all rounds of every session, a bankruptcy occurred: in the late periods of every round money invested by players was used to pay interest (i.e., the outcome was efficient). ${ }^{6}$ In this respect, the outcome in the laboratory is no different than that in Massachusetts in 1920 or Albania in 1997. An important difference, however, is that the laboratory allows us to carefully study the development of the Ponzi scheme and the individual choices underlying it.

Next, we consider the decision to enter the scheme. Rationally, there is no reason to enter later than period 1 (cf. ESM Appendix A). Indeed, it is optimal to enter in period 1 , because in all treatments the amount available in IF is sufficient to pay interest in at least two periods $\left(t_{p}=2 \vee t_{p}=4\right.$ in all treatments; cf. ESM Appendix B). Out of the 1792 individual participations in Ponzi games that we observed (224 participants, 8 rounds each), only $2(0.1 \%)$ did not invest in period 1 . Hence, 'late' entry plays no role in our data.

We continue with an overview of the periods in which investors withdraw. For this purpose, Table 2 provides an overview of median withdrawal periods across treatments. This shows that a higher interest rate has the predicted effect of reducing the time spent in the investment fund. Moreover, the uninformed withdraw earlier when there are more informed than when there is only one informed. Statistical tests for treatment comparisons are provided below.

An overview of aggregate participation can be visualized using survival functions, i.e., the fraction over time of subjects investing in the scheme. Figure 1 displays the raw data in this way.

\footnotetext{
${ }^{6}$ It is not a priori obvious that they will collapse, because participants could withdraw their investment before $\chi$ has been depleted.
} 


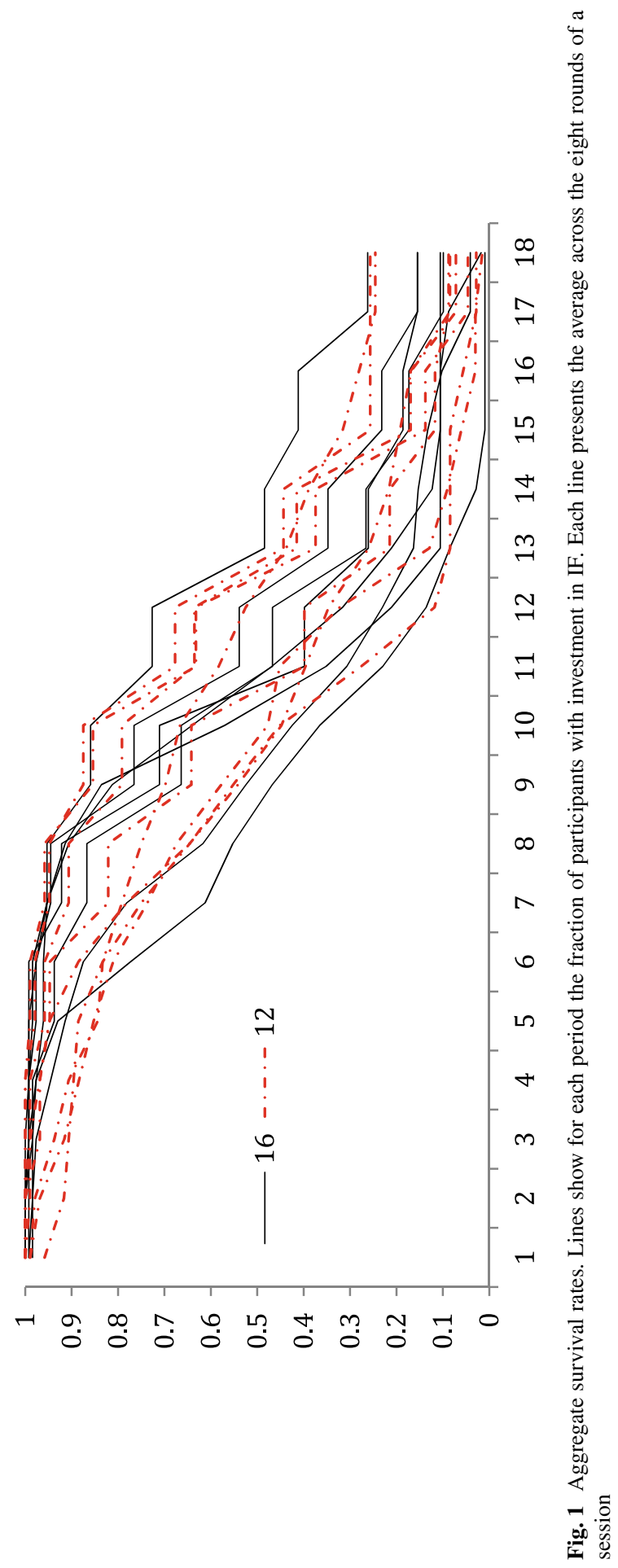


To make all sessions directly comparable, in this presentation of the data, we rescaled time by doubling the survival time of the sessions with a high-interest rate. This rescaling treats each investment decision as if it lasts for two periods. With this adjustment, for all sessions, the quasi-symmetric equilibrium derived above is for everyone to withdraw in period 12 in case of 1 informed player and for all uninformed (and if $\chi$ is small, also the informed) to leave in period 8 when there are 6 or 8 informed players (cf. ESM Appendix B). Figure 1 shows no such step function. Instead, investors start withdrawing as soon as investing becomes risky (period $t_{p}=4$ ), while many keep their money invested even when it is certain that interest is being paid out of subjects' investments. This observation is not a consequence of aggregating across rounds with varying $\chi$ values. We observe the same in individual rounds. Nevertheless, the major decline in investment takes place between periods 8 and 12 in all sessions. Hence, in aggregate, behavior approximates that in equilibrium. $^{7}$

\subsection{Treatment effects}

Result 1 The number of investors does not affect behavior.

Table 2 and Fig. 1 suggest that the number of subjects does not affect participation. This is confirmed by a Renyi test $(Q=2.41, p=0.12$, obs $=16) .{ }^{8}$ This supports prediction (1) in the previous section. Therefore, from here onward, we pool our data for $N=12$ and $N=16$ and continue the analysis of the other two treatment variations (interest rate and number of informed).

Result 2 An increase in the number of informed investors reduces the duration of investment by the uninformed.

Figure 2 shows the average survival functions per treatment (see ESM Appendix $\mathrm{D}$ for the functions split per session). Following result 1, we aggregate across $N=12$ and $N=16$. Each line then shows the average across four sessions. The two black lines (solid and dashed) compare the low interest sessions with 1 and 8(6) informed investors. The grey lines do the same for the high-interest sessions.

Investors appear to keep their money in IF longer when there is 1 informed investor than when there are 8(6). Using a two-sample Renyi test we (marginally) reject the null of no effect for both cases $(Q=7.23, p<0.01$, obs $=8$, for low interest;

\footnotetext{
7 Figure 1 truncates observations beyond period 18. Often, the fund remained 'alive' longer. In some cases, two or three investors tacitly colluded and stayed in until bankruptcy occurred when the fund could no longer pay interest.

8 The Renyi test is the analogue of a Kolomogorov Smirnov test that corrects for censored data. 'obs' refers to the number of uncensored observations, taking the session as the unit of observation. This gives eight observations per treatment. A power analysis indicates that 36 observations per treatment would be needed to obtain a power of $80 \%$. The test is therefore underpowered. The actual difference, however, is small in size. If we were to treat each individual as an independent observation (increasing the power to over 99\%), the difference would still be insignificant $(Q=1.86, p=0.13$, obs $=1.367)$. We are therefore confident of the robustness of this result. Similarly, we find no evidence of a group size effect when conducted all tests reported below for our other two treatments separately for $N=12$ and $N=16$.
} 


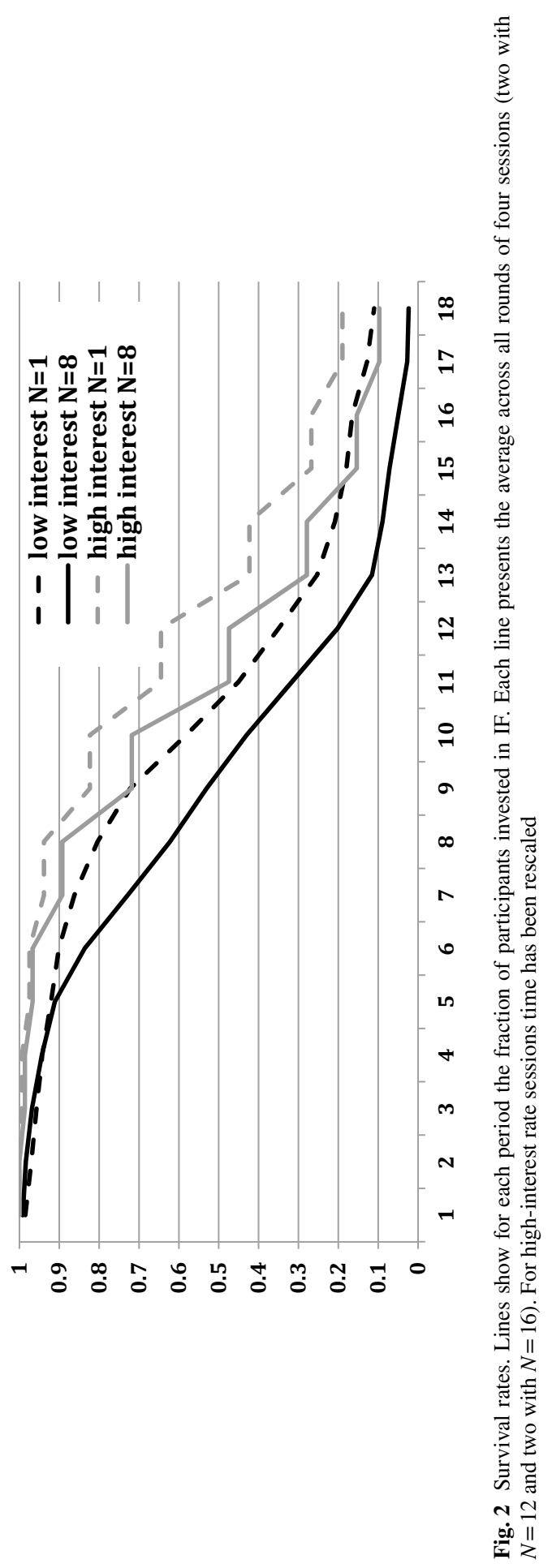


$Q=6.30, p<0.08$, obs $=8$, for high interest). ${ }^{9}$ This confirms comparative static prediction (2).

Result 3 The duration of investment is lower when $r=0.2$ than when $r=0.1$.

To compare the high and low interest sessions, first, consider the situation without rescaling time for the high-interest sessions. There is then a very strong decrease in survival time. For both 1 and 8(6) informed investors, Renyi tests confirm the result $(Q=15.71, p<0.01$,obs $=8$, for 1 informed investor; $Q=16.16, p<0.01$, obs $=8$, for 8(6) informed investors). This confirms comparative static result (3).

As discussed above, rescaling is predicted to equalize the investment duration in the high and low interest cases. Figure 2 allows for this comparison by considering the two dashed lines $(N=1)$ or the two solid lines $(N=8)$. In both cases, higher interest appears to yield longer survival. Renyi tests show that the difference is significant for both one $(Q=4.68, p<0.01$, obs $=8)$ and eight informed $(Q=5.15, p<0.01$, obs $=8)$. Hence, a higher interest is making subjects stay in longer after time rescaling. Even though the higher interest increases the per-period payoff, it also increases the probability of a bankruptcy. With our parameters, these two effects should cancel out in equilibrium, but apparently the higher interest makes our subjects take more risks.

Finally, ESM Appendix E provides evidence of learning. Specifically, there is lower variance in the survival functions of periods 5-8 than in periods $1-4$.

\subsection{Investment decisions}

To better understand these results, we describe the observations in each session with a discrete (proportional) hazard model of the decision to withdraw money (e.g., Lancaster 1990, or Cameron and Trivedi 2005). This model describes the probability of withdrawing in period $t$ conditional on participating in $t-1$. This hazard rate is a function of the period and of a set of covariates, $Z$ (which may or may not be time dependent). We apply a parameterization using the exponential distribution. ${ }^{10}$ The hazard rate $\theta_{t}$ is given by:

$$
\theta_{t}=1-\exp \left(-\exp \left(\gamma_{t}+\beta^{\prime} Z\right)\right),
$$

where $\gamma_{t}$ is a baseline hazard that may vary across periods. When estimating the coefficients $\gamma_{t}$ and $\beta$, we correct for censored data (subjects cannot participate after bankruptcy, even though they would have chosen to).

We estimate three versions of the model. In each, we rescale time in the highinterest sessions as described above. The models differ in the group of subjects involved and the variables used for $Z$. In model 1, we study the basic characteristics in the structure of individual behavior. This model uses all choices (across 8

\footnotetext{
${ }^{9}$ Here (and in the tests for Result 3 ) we take a conservative approach and treat the mean across individuals and rounds as the unit of observation.

${ }^{10}$ Equation (1) gives the continuous function underlying the decisions, which are only observed at disjoint time intervals. See, e.g., Lancaster (1990) or Cameron and Trivedi (2005) for details about the step from continuous time to discrete time intervals.
} 
rounds) by all participants. $Z$ consists of two variables, one indicating the player type (informed or uninformed) and the other the $\chi$ drawn. The latter is set to zero for the uninformed.

Models 2 and 3 test particular equilibrium predictions. Model 2 considers only the choices of the uninformed and model 3 only the choices of the informed. One characteristic of the equilibrium is that the uninformed do not react to the investment decisions of the informed ('herding') unless the latter withdraw before $t^{U}$, which will not happen in equilibrium. Model 2 investigates herding. Here, $Z$ (now time dependent) only consists of a variable describing the fraction of informed investors that withdrew in the previous period. In model 3 we test the prediction that the hazard rate of the informed will be different when $t_{\chi}<t^{U}$ than when the reverse holds. For this purpose $Z$ consists of two variables, $\chi_{1}$ and $\chi_{\mathrm{h}} \cdot \chi_{1}=\chi$ if $t_{\chi}<t^{U}$, and $\chi_{1}=0$, otherwise. $\chi_{\mathrm{h}}=\chi$ if $t_{\chi}>t^{U}$, and $\chi_{\mathrm{h}}=0$, otherwise. Our equilibrium prediction is that there will be no effect of $\chi_{1}$ (the informed stay in until $t^{U}$, irrespective of $\chi$ ) and a negative effect of $\chi_{\mathrm{h}}$.

Note that individual choices within a session are not independent observations. One way to deal with this is to estimate the models using all observations while clustering at the session level. Treatment effects can then be tested with dummies interacting specific treatments with the variables of interest. We follow this approach in ESM Appendix F. Here, we apply a method that more directly fits the treatment structure of our experimental design. We estimate the models for each session separately. We then use the estimated coefficients as summary statistics for behavior in the session. We believe that the hazard coefficients are more informative statistics than, for example, the median period of withdrawal (cf. Table 2), because the structure of behavior is most accurately described by a hazard rate. ${ }^{11}$ We thus use the estimated coefficients to summarize the data per session. Because we are interested in the treatment comparisons, we will not discuss statistical properties of the estimated hazard coefficients per se. Instead, we compare the coefficients across (groups of) sessions.

For each model, we have a set of estimated coefficients per session. This gives for each treatment cell four independent observations per coefficient. The average (across sessions) values of the coefficients estimated for model 1 are given in ESM Appendix G. The results show that the conditional probability of withdrawing increases over time. Moreover, ceteris paribus, the informed are more likely to withdraw first. This counterintuitive result may be due to the fact that (as we will see below) the uninformed closely follow the behavior of the informed. The structure of the game is such that there is a one period delay between the moment an informed withdraws and the moment this is noticed by the uninformed. Finally, the estimates for model 1 show the intuitive result that the informed stay in longer when there

\footnotetext{
11 Our approach has the advantage of not imposing assumptions about the error terms across treatments. Unless indicated otherwise, our results are robust to using the method of ESM Appendix F. By and large, our conclusions find slightly stronger support using the method in ESM Appendix F than in the main text.
} 
Table 3 Herding by the uninformed

\begin{tabular}{|c|c|c|c|c|c|c|c|c|}
\hline & Lo 1 & Lo 1 & Lo 1 & Lo 1 & Lo 8 & Lo 8 & Lo 8 & Lo 8 \\
\hline Herd & 0.221 & $--^{\mathrm{a}}$ & 1.906 & -0.474 & 4.347 & 3.063 & 3.281 & 2.477 \\
\hline Average & 0.55 & & & & $3.29 * * *$ & & & \\
\hline$\chi_{1}$ & -0.866 & -2.420 & -0.417 & -1.222 & -1.304 & -0.298 & -0.974 & -0.731 \\
\hline Average & $-1.23^{*}$ & & & & $-0.83^{* *}$ & & & \\
\hline$\chi_{\mathrm{h}}$ & & & & & -1.606 & -0.945 & -2.016 & -0.967 \\
\hline \multirow[t]{2}{*}{ Average } & & & & & $-1.38 * *$ & & & \\
\hline & Hi 1 & Hi 1 & Hi 1 & Hi 1 & Hi 8 & Hi 8 & Hi 8 & Hi 8 \\
\hline Herd & -0.409 & 1.066 & 0.345 & 1.342 & 0.400 & 0.549 & 4.594 & 4.221 \\
\hline Average & 0.59 & & & & 2.44 & & & \\
\hline$\chi_{1}$ & -0.618 & -0.670 & -0.417 & -0.902 & -0.331 & -0.607 & -0.355 & -1.010 \\
\hline Average & $-0.65 * * *$ & & & & $-0.58 * *$ & & & \\
\hline$\chi_{\mathrm{h}}$ & & & & & -0.879 & -0.701 & -1.108 & -0.915 \\
\hline Average & & & & & $-0.90 * * *$ & & & \\
\hline
\end{tabular}

Coefficients are estimated per session, using data from all rounds from only the uninformed for the variable Herd and the informed for $\chi_{1}$ and $\chi_{\mathrm{h}}$. Numbers give the coefficients for Eq. (1) of the variable depicted in the first column in the various sessions. Variables are defined in the main text

$* / * * / * * *$ Indicates that the average coefficient is statistically significantly different than $0(t$ test $)$ at the 10-/5-/1\%-level

${ }^{a}$ No coefficient is estimated, because the (one) informed always withdrew so early that no uninformed followed

is more external money. Whether the behavior of the informed is in line with our benchmark equilibrium is tested in more detail in model 3, below.

In ESM Appendix G, we also use the estimates of model 1 for an additional test of treatment effects. We find, i.a., that the significant differences between high and low interest sessions are observed for the 'risky' periods 5-8 and 9-12. Though not rational (because time rescaling equalizes the risks), this is somewhat intuitive; it indicates that high-interest payments make it more attractive to risk facing bankruptcy, because the immediate earnings via interest are higher. Similarly, there is a significant difference between 1 and 8 informed in the periods beyond 12, where it is certain that interest is being paid out of participants' investments. To better understand such results, we consider both types separately. First, we turn to model 2 and focus specifically on the choices of the uninformed.

Model 2 tests whether herding takes place. When estimating, we again correct for censored data. The estimation results per session are presented in Table 3. The "Herd" rows show the coefficients addressing the herd behavior discussed above.

The positive numbers observed in 13 of the 15 sessions, where we have estimates indicate herding. Hence, the uninformed seem more likely to withdraw if (some of) the informed did so previously. This is only the case, however, when there are multiple informed participants. A $t$ test of the seven observations with one informed 
cannot reject the hypothesis that the mean coefficient is equal to zero $(p=0.14) .^{12}$ For the eight observations with eight (six) informed, a $t$ test does reject the null hypothesis that the mean coefficient is zero $(p<0.01)$. The fact that the effect is stronger when there are eight (six) informed than when there is only one is supported by a Mann-Whitney test $(p<0.01$, obs $=15)$. All in all, our results provide support for herding behavior by the uninformed, but only when there are sufficiently many informed participants to follow. ${ }^{13}$

Finally, model 3 tests the prediction that the informed will (not) adjust to variations in $\chi$ when $t_{\chi}$ is high (low). We test this by introducing the variables $\chi_{1}$ and $\chi_{\mathrm{h}}$ described above. Table 3 (the rows " $\chi_{1}$ " and " $\chi_{\mathrm{h}}$ ") presents the results for these variables per session. We note that for the sessions with one informed, the parameters imply that $t_{\chi}<t^{U}$, for all $\chi$ (cf. ESM Appendix B). Hence, there are no observations for $\chi_{\mathrm{h}}$ in this treatment.

We observe that all of the 16 coefficients for $\chi_{1}$ (where the predicted value is zero) and all of the 8 coefficients for $\chi_{\mathrm{h}}$ (predicted to be negative) are negative. ${ }^{14}$ Contrary to rational reasoning, the result for $\chi_{1}$ shows that the informed respond to lower values of $\chi$ by a higher conditional probability of leaving the scheme even when $t_{\chi}<t^{U}$. This means that they do not expect the uninformed to stay in until the period that maximizes their expected earnings.

\section{Conclusions}

Ponzi schemes can have major consequences for the well-being of those involved. A better understanding of why they occur, their consequences, the role of informed insiders, and how they may be avoided in the future requires extensive theoretical and empirical research. In our laboratory experiment, the Ponzi schemes work similar to those in the outside world. Our experimental data thus allow us to get a first grasp at behavior in Ponzi schemes. It appears that our quasi-symmetric equilibrium captures the comparative statics reasonably well. As the model predicts for our parameterization, we find that participation duration (1) is unaffected by the number of investors; (2) decreases with the relative number of informed investors; and (3) decreases with the interest rate.

Nevertheless, there are features of the data that are not predicted by our benchmark. This may be due to the assumed risk neutrality. Even relaxing this assumption, however, will leave us with the observation of investments in periods when everyone can be certain that the initial investment $\chi$ has been completely depleted. This suggests that a model assuming common knowledge of rationality cannot explain the

\footnotetext{
12 We note that the power of this test $(0.39)$ is low. Importantly, as reported in the main text we do find that mean herding is significantly lower with one informed than with $8(6)$.

13 The results in ESM Appendix F also show significant herding when there is one informed.

14 The corresponding coefficients in the regression model of ESM Appendix F are all significantly negative.
} 
data. As argued by Tirole (1982), it is simply not rational to participate in a Ponzi scheme (in our case, after a certain amount of time), but people do.

An important conclusion from our data is that the distinction made between informed and uninformed investors matters. Contrary to our benchmark equilibrium, we find that the uninformed investors follow the movements of the informed and can, therefore, potentially be taken advantage of. Rationally, the uninformed should consider that it is in the interest of the informed to mislead them. The fact that the uninformed can be misled in this way is often considered a major reason for the occurrence of such schemes (Sadiraj et al. 1997; Sadiraj 1999; or Bhattacharya 2003).

Our results provide a dilemma to the informed wanting to start such a scheme. On the one hand, they would like to restrict the information to a 'happy few'. On the other hand, our results show that an increase in their number will make the uninformed more susceptible to their behavior and, therefore, will increase the potential for making money off of them.

We believe that our experiments provide interesting insights into behavior in Ponzi schemes. Nevertheless, they also leave many important questions unanswered. For example, it would be interesting to see whether experienced subjects are less likely to lose money in these schemes. More fundamentally, we have not addressed the possibility of starting such schemes, or even of different participants starting competing schemes. In our view, this is an interesting topic deserving future attention.

Open Access This article is distributed under the terms of the Creative Commons Attribution 4.0 International License (http://creativecommons.org/licenses/by/4.0/), which permits unrestricted use, distribution, and reproduction in any medium, provided you give appropriate credit to the original author(s) and the source, provide a link to the Creative Commons license, and indicate if changes were made.

\section{References}

Bhattacharya, U. (2003). The optimal design of Ponzi-schemes in finite economies. Journal of Financial Intermediation, 12, 2-24.

Brock, W. (1982). Asset prices in a production economy. In J. Green (Eds.), The economics of information and uncertainty. Chicago: University of Chicago Press.

Brown, M., Trautmann, S. T., \& Vlahu, R. (2012). Contagious bank runs: Experimental evidence; DNB Working Paper 363, Amsterdam.

Cameron, A. C., \& Trivedi, P. L. (2005). Macroeconometrics: Methods and applications. Cambridge: Cambridge University Press.

Gërxhani, K., \& Schram, A. (2009). Clientelism and polarized voting: Empirical evidence. Public Choice, 141, 305-317.

Gilles, C., \& LeRoy, S. (1992). Bubbles and charges. International Economic Review, 33, 323-339.

King, R., Smith, V., Williams, A., \& van Boening, M. (1993). The robustness of bubbles and crashes in experimental stock markets. In Richard H. Day (Eds.), Nonlinear dynamics and evolutionary economics (pp. 183-200). Oxford: Oxford University Press.

Lancaster, T. (1990). The econometric analysis of transition data. Cambridge: Cambridge University Press.

O’Connell, S., \& Zeldes, S. (1988). Rational Ponzi-schemes. International Economic Review, 29, $431-450$. 
Sadiraj, K. (1999). Albania: Transition to a market economy; PhD-thesis Tinbergen Institute, University of Amsterdam.

Sadiraj, K. S., van Wijnbergen, \& van Ewijk, C. (1997). The Albania crisis: Pyramid schemes and their economic and political effects, mimeo, University of Amsterdam.

Shala, A. (1997). The Albanian Crisis; mimeo, Institute of Transnational Economies.

Shotter, A., \& Yorulmazer, T. (2009). On the synamics and severity of bank runs: An experimental study. Journal of Financial Intermediation, 5, 409-423.

Smith, V., Suchanek, G., \& Williams, A. (1988). Bubbles, crashes, and endogenous expectations in experimental spot asset markets. Econometrica, 56, 1119-1152.

Sunder, S. (1995). Experimental asset markets: A survey. In J. Kagel (Eds.), Handbook of experimental economics. Princeton: Princeton University Press.

Tirole, J. (1982). On the possibility of speculation under rational expectations. Econometrica, 50, 1163-1181.

Tirole, J. (1985). Asset bubbles and overlapping generations. Econometrica, 53, 1499-1528.

van Brabant, J. M. (1998). Political economy of transition: Opportunities and limits of transformation. London: Routledge. 